\title{
Maximum Inflated Stent Diameter
}

National Cancer Institute

\section{Source}

National Cancer Institute. Maximum Inflated Stent Diameter. NCI Thesaurus. Code C150197.

Maximum diameter to which a stent can be distended. 